\title{
APUNTES SOBRE UNA AGENDA PARA LA MODERNIZACIÓN DE LA UNIVERSIDAD DE COSTA RICA: CONTEXTO Y PRIORIDADES
}

\author{
Flora Eugenia Salas Madriz
}

Resumen: En este artículo se bace un análisis coyuntural de la educación superior pública costarricense, de frente al contexto bistórico de las décadas del los años ochenta y los noventa, en donde se da un proceso de reversión del Estado Benefactor.

Se propone una revisión profunda de las bases filosóficas $y$ administrativas de la universidad pública en general, $y$ de la Universidad de Costa Rica en particular, para culminar con una agenda de prioridades para la modernización de la gestión universitaria, a fin de que el proyecto de educación superior pública emprendido por la U.C.R. hace ya más de cuarenta años, se ajuste a los nuevos requerimientos de la economía, la cultura y la sociedad del presente siglo.
Dos grandes crisis han sido diagnosticadas en la educación superior latinoamericana: la del crecimiento cuantitativo, que se dio aproximadamente entre los años 1967 y 1981, producto de las demandas del sistema económico de corte desarrollista, de sustitución de importaciones (Jofré, 1994), y la de calidad (Tünnermann, 1996); crisis que se ha hecho cada vez más aguda, consecuencia de la masificación producto de la explosión cuantitativa, de los problemas de financiamiento generados por la aplicación de los Programas de Ajuste Estructural, y del bajo rendimiento, tanto académico como pedagógico, debido a la obsolescencia del modelo educativo tradicional.

En ambas crisis el Estado ha desempeñado un papel fundamental. En el primer caso, el Estado de corte benefactor o providencial como potenciador del impacto social de la educación y la seguridad social; en el segundo, la reducción del mismo, con su respectivo retiro progresivo de la ejecución de programas de interés social, específicamente, de la educación.

Luego de la profunda crisis de crecimiento que se experimentó entre las décadas de los años setenta y ochenta, en la actualidad se ha desarrollado una amplia discusión en torno a la crisis generalizada de la educación, particularmente en lo que respecta a la calidad, como un fenómeno común en América Latina. Esta profunda crisis 
ha tenido un fuerte impacto en áreas vitales del ordenamiento económico, político y cultural latinoamericanos.

Hoy, por ejemplo, es ampliamente conocido en la región centroamericana el impacto en la educación, de la implementación de los Programas de Ajuste Estructural propuestos por el Banco Mundial, en la década de los años ochenta, orientados a una reorganización de los factores productivos de los países, a fin de hacer sus economías más eficientes. Esta reorganización implicó una reestructuración a nivel macroeconómico que tuvo como principal objetivo la reforma del Estado y la redefinición de las políticas sociales.

La principal tesis para la implementación de los Programas de Ajuste Estructural fue el planteamiento de que el Estado de corte benefactor introdujo, paulatinamente, una serie de obstáculos y contradicciones en la lógica del mercado nacional e internacional, que habían tenido como consecuencia una baja productividad, poco estímulo a las inversiones privadas, uso inadecuado de recursos humanos, físicos y financieros, y escasos controles, debido a la alta politización implícita en los procesos de toma de decisiones gubernamentales. El principal elemento a considerar fue, con base en lo anterior, el criterio de racionalidad económica. La eficiencia, la eficacia y la calidad, se identificaron como características esenciales de economías saludables. La medida terapéutica del Banco Mundial se encaminó al logro de una racionalización del gasto público, el ajuste estructural del mercado financiero, la reducción del proteccionismo, y la estimulación de los sectores y mercados privados a nivel nacional (Arroyo, J.A. et al., 1992: 7).

El proceso de reestructuración del Estado, introdujo en nuestros países, a nivel general, una serie de problemas nuevos relacionados con la redefinición de lo público y con el traslado de actividades asumidas en el pasado por el Estado, al sector privado. Esto tuvo efectos positivos y negativos. Por una parte, se ha logrado una relativa estabilización a nivel macroeconómico, al menos en lo que respecta a deuda externa, acompañada de una mayor diversificación económica; por otra, esta estabilización ha tenido un enorme costo social y político, pese a la introducción de la noción de Ajuste Estructural con Rostro Humano, propuesta por la UNICEF, que planteaba que el ajuste debía generar estabilidad y crecimiento económico, garantizando la satisfacción de las necesidades básicas de la población (Arroyo, J.A et al., 1992: 10).

Los programas de ajuste estructural implicaron un cambio de modelo económico y estatal. Este cambio de modelo, en ambos casos, se ejecutó en Costa Rica a través de reformas económicas orientadas a la estabilización macroeconómica, la liberalización financiera y la apertura de mercados; así como de procesos de privatización, redefinición de funciones, desregulación y descentralización estatales.

La reforma estatal trajo consigo una redefinición de la gestión pública, que se basaba en la introducción de la racionalidad económica empresarial privada. La eficiencia, la eficacia y la calidad, se convirtieron en derroteros de la administración pública. Por este motivo, la primera tesis que se plantea es que las instituciones públicas en general, y la universidad en particular, enfrentan una crisis de modelo institucional y de organización, debido a que no se ha logrado hacer compatibles las estructuras centralizadas, concentradoras en la toma de decisiones, burocráticas, y altamente proclives a la politización, con una gestión coherente con los postulados de eficiencia, eficacia y calidad. Como consecuencia de esta tesis, el primer punto para una agenda de modernización de la universidad pública, consiste en un replanteamiento de su misión-visión, y en una reestructuración organizacional, donde la participación y la concertación de los distintos actores universitarios, así como de la sociedad y del Estado, sean posibles. Por esto, se considera que es el modelo de universidad pública lo que realmente está en crisis; y que las crisis colaterales en los ámbitos de la cali- 
dad, presupuestario y de gestión, así como la agudización de los problemas pedagógicos, obedecen, básicamente, a la primera.

Los falsos problemas nos llevan a falsas respuestas. El esfuerzo por modernizar la universidad pública, en el caso particular de la Universidad de Costa Rica (en adelante U.C.R.), que es la más antigua y consolidada del país, ha iniciado algunos procesos de cambio, desde una lógica administrativa tradicional, de corte "de arriba hacia abajo". Desde la teoría administrativa moderna, el cambio sin participación real no es posible. De hecho, una de las universidades públicas menos democráticas es la U.C.R., debido a que la estructura organizacional excluye "legalmente", de la toma de decisiones a amplios sectores de la comunidad universitaria, entre los que destaca el sector docente. Para explicar lo anterior, hay que hacer un breve recorrido sobre las políticas implementadas en los últimos años en la contratación del personal docente interino.

El docente interino, dada su condición, no puede participar en la toma de decisiones en Asambleas de Escuela, de Facultad o de Sede. Tampoco tiene derecho a voto, aunque tenga derecho a vOz, caso de que así lo permita la instancia legitimada por el Estatuto Orgánico. Además de una contratación generalizada de jornadas de un cuarto de tiempo e, incluso, inferiores, y del no pago de períodos interlectivos, esta situación resulta particularmente grave, si se toma en cuenta que los docentes interinos contratados, superan en un $25 \%$ al personal docente propietario, como muestra la siguiente tabla de distribución de personal docente por tipo de nombramiento, durante el período 1991-1995. Esto refleja la realidad del clima organizacional de la U.C.R. Asimismo, el hecho de que cada vez menos personas participen en la toma de decisiones, atenta directamente contra el principio de autonomía universitaria, en el tanto que obstaculiza e, incluso, impide verdaderos mecanismos de participación crítica y creativa al interior de la comunidad universitaria misma. Si la autonomía universitaria no opera en su totali- dad, la institución universitaria se desvirtúa y se convierte en un espacio de impunidad, donde es fácil que haya predominio de intereses de grupos de presión internos y externos a la universidad, que obstaculizan el cambio.

Tabla No. 1

Distribución del personal docente por tipo de nombramiento Entre 1991 y 1995

\begin{tabular}{|c|c|c|c|c|}
\hline \multirow[t]{2}{*}{ Ciclo } & \multirow[t]{2}{*}{ Total } & \multicolumn{2}{|c|}{$\begin{array}{c}\text { Tipo } \\
\text { Nombramiento }\end{array}$} & \multirow{2}{*}{$\begin{array}{c}\text { Razón } \\
\text { Interino/ } \\
\text { Propietario }\end{array}$} \\
\hline & & Propiedad & Interino & \\
\hline $1-91$ & 4.151 & 1.848 & 2.303 & 1,25 \\
\hline $2-91$ & 4.307 & 1.906 & 2.401 & 1,26 \\
\hline $1-92$ & 4.296 & 1.918 & 2.378 & 1,24 \\
\hline $2-92$ & 4.446 & 1.971 & 2.475 & 1,26 \\
\hline $1-93$ & 4.460 & 1.900 & 2.560 & 1,35 \\
\hline $2-93$ & 4.304 & 1.916 & 2.388 & 1,25 \\
\hline $1-94$ & 4.376 & 2.003 & 2.373 & 1,18 \\
\hline $2-94$ & 4.179 & 1.858 & 2.321 & 1,25 \\
\hline $1-95$ & 4.077 & 1.789 & 2.288 & 1,28 \\
\hline $2-95$ & 3.742 & 1.667 & 2.075 & 1,24 \\
\hline TOTAL & 42.338 & 18.776 & 23.562 & 1,25 \\
\hline
\end{tabular}

Fuente: Adaptado de: Universidad de Costa Rica (1995). Serie de diagnósticos. Panorama cuantitativo. Oficina de Planificación Universitaria, p. 48.

Esta profunda lesión a la autonomía, refleja cuánto se ha distanciado la gestión universitaria de su misión primordial de velar por la transformación social orientada al logro del bien común, promoviendo un régimen democrático, donde las decisiones personales y colectivas pueden ser tomadas, declaradas y realizadas, con plena libertad, como se estipula en el Estatuto Orgánico (Título I, artículos 1, 2 y 3).

Otro de los grupos excluidos de la participación libre y crítica en la toma de decisiones es el estudiantado, debido a que no se cuenta en la vida universitaria con espacios oportunos y suficientes para propiciar la participación activa de los estudiantes en la toma de decisiones. Precisamente, una gran ausente en las políticas universitarias es la evaluación estudiantil del desempeño docente-administrativo. Sus necesidades, intereses y críticas son 
escasamente considerados cuando se toman decisiones en torno a aspectos de índole docente, académica o administrativa.

Además de la exclusión de estos sectores de la comunidad universitaria, la sociedad no es informada ni consultada sobre el desempeño de la universidad. Por esto, otro de los problemas urgentes que debe resolver la universidad pública, es su vinculación con la sociedad y con el sector productivo. La administración burocrática debe ser reemplazada por una gestión estratégica, que permita rearticular y redefinir el rol de la universidad pública al interior de la realidad contemporánea, donde la globalización y el paradigma neoliberal dictan las pautas económicas mundiales; pautas a las cuales, evidentemente, no podemos sustraernos.

El nuevo rol de lo público está mediado por la dinámica de la gestión privada; por ello, erróneamente se ha pretendido resolver los apremiantes problemas de la gestión pública actual, implementando estrategias de gestión empresariales, sin haber hecho los ajustes organizacionales, legales y políticos necesarios para que tales estrategias sean constructivas y no disfuncionales. En este sentido, se coincide con Juan Carlos Tedesco (1991), cuando señala que la universidad pública es un asunto de supervivencia en América Latina, y que su principal reto consiste en crear condiciones propicias para una gestión dinámica e innovadora, con fácil respuesta al cambio y a las nuevas demandas sociales; todas estas, características propias de la gestión privada.

El problema de la calidad está asociado al modelo de universidad pública, a la estructura organizacional, a las estrategias de gestión y a la capacidad de respuesta de las autoridades universitarias ante el hecho de que el cambio es ineludible. En este sentido, ver el problema de la calidad como un asunto aislado, no permite desarrollar políticas viables y adecuadas para superar la crisis. La principal crisis está, por esto, en el manejo mismo de la crisis (Offe, 1990). No pueden visualizarse los problemas como asuntos ajenos a la universidad, sino como consecuencia de su estructura misma, alterada por el nuevo juego contemporáneo entre las características y los roles de lo público y lo privado.

\section{El nuevo rol de la universidad pública: ¿hacia dónde se puede y se debe ir?}

Las estrategias de gestión privadas son insuficientes para el logro de los objetivos de políticas de carácter público. Si bien es cierto, en la actualidad los bienes públicos y privados no deben diferir en lo que respecta a calidad, sí deben diferenciarse claramente en cuanto a destinatarios, fines y énfasis estratégico. Parece que esta es una perogrullada; sin embargo, se considera que aquí es donde se presenta el mayor grado de confusión. La orientación de la gestión privada es la competitividad; la de la gestión pública, el desarrollo nacional. Por esto, como falsamente se ha creído en algunas universidades públicas costarricenses, la universidad privada no es una amenaza, en el sentido que ésta se orienta a satisfacer demandas sociales orientadas privadamente, y no demandas sociales orientadas por el interés social.

La universidad pública debe encauzar su quehacer a la atención permanente, eficaz, eficiente, y de alta calidad, de la demanda social para satisfacer los requerimientos personales, sociales, y empresariales del nuevo orden económico nacional y mundial. En este sentido, no toda demanda del mercado está orientada al desarrollo nacional y viceversa. Esto es particularmente cierto en los países subdesarrollados, debido a que muchas de las áreas educativas estratégicas para el desarrollo nacional, por su costo de inversión, tasa de retorno, complejidad organizacional y rentabilidad a corto plazo, no pueden ser asumidas por el sector privado con el nivel de calidad requerido. Un típico ejemplo de esta realidad es la investigación a nivel general, y la formación de posgrado en áreas estratégicas como las ciencias naturales y de la salud, 
y las ingenierías. En este sentido, retomando a Tedesco, la universidad pública constituye un problema urgente de supervivencia para los países pobres (Tedesco, 1991: 221).

Se considera indispensable que la universidad pública asuma su rol, tomando el sector terciario privado no como modelo, sino como complemento. La universidad privada satisface una serie de demandas sociales que pueden ser asumidas a nivel empresarial; pero esto no significa que la única función del sector educativo terciario es satisfacer la demanda de mercado. Al respecto, la universidad pública debe orientar iniciativas globales de calidad, que incluyan el sector terciario privado; y no, como lo ha hecho hasta ahora, orientándose por las estrategias privadas como sustituto de una verdadera modernización del sector educativo terciario público.

La falta de claridad en lo que hoy significa público y privado ha hecho que la sobrecarga de demandas sociales sobre el Estado, haya generado una crisis que va más allá de lo puramente estructural y funcional, para convertirse en crisis política y, por ende, en una pérdida creciente de legitimidad del sector público ante la sociedad (Offe, 1990: 119 y ss.). El modelo político democrático contemporáneo se encuentra en crisis, debido a la incoherencia entre el aumento de demandas sociales sobre el Estado, y la capacidad formal y real de éste para responder a tales demandas (Offe, 1990). Como principal contradicción, la gestión de instituciones públicas sufre la dicotomía entre políticas participativas y democráticas, y gestión técnica. Cuando prima lo técnico, se pierde la naturaleza social del servicio o producto público; cuando prima lo político, se pierde su naturaleza económica.

A manera de síntesis de lo expuesto hasta aquí, la obsolescencia del proyecto económicopolítico que sustentaba el modelo de universidad latinoamericana consolidado en el período desarrollista, produce serias disfunciones y distorsiones para el nivel de desempeño y el nuevo rol de las instituciones universitarias públicas tradicionales. Al pretender realizar los ajustes sobre la marcha de la presión social entre lo público y lo privado, sin que exista un nuevo modelo de universidad coherente con los actuales requerimientos del sistema económico basado en el uso intensivo del conocimiento, sólo se corre el riesgo de desgastar aún más la legitimidad de la institución para condenarla, finalmente, a su extinción.

Es un hecho que la tendencia que ha ganado más terreno en Latinoamérica es la fórmula "menos Estado, más iniciativa privada". Sin embargo, aún no está claro qué actividades anteriormente asumidas por el Estado siguen siendo estratégicas para orientar el desarrollo nacional. Precisamente, un ejemplo de esta confusión entre interés privado e interés público, es el I.C.E., institución estratégica en el tanto que las telecomunicaciones son hoy condición necesaria para un adecuado desempeño de la economía nacional, dentro de la lógica de la economía internacional. Un país pequeño con un sistema de telecomunicaciones sólido y altamente desarrollado, orientado por el interés público, constituye una ventaja competitiva fundamental, ya que la información es hoy uno de los principales elementos de la actividad económica. En este sentido, no es lo mismo privatizar la Fábrica Nacional de Licores que el Instituto Costarricense de Electricidad.

Si el conocimiento es hoy el principal factor productivo, parece obvio que en nuestros países la universidad es estratégica para el desarrollo desde una perspectiva de sostenibilidad; la cual, necesariamente, involucra el mejoramiento de la calidad de vida de la población, a través de un sistema educativo público moderno, eficiente y de alta calidad.

\section{Condiciones para el cambio: un nuevo modelo de universidad pública}

Las ventajas que disfrutaron docentes y administrativos en las décadas de los años sesenta y setenta, cuando hubo un importante impulso económico por parte del Estado para las universidades públicas 
debido a los intereses del modelo desarrollista, se fueron restringiendo en forma creciente en la década de los años ochenta, para extinguirse prácticamente en la de los noventa. La universidad pública se convirtió en un fuerte peso económico para el Estado, y la realidad financiera cambió radicalmente, de solvente a deficitaria. Lentamente, los derechos del pasado se trastocaron privilegios para quienes los habían ganado en ese período, y la época de solvencia y de "recursos y oportunidades a manos llenas", llegó a su fin.

En el período de auge, era relativamente sencillo para los docentes de nuevo ingreso, encontrar estabilidad laboral, y oportunidades de becas para estudios de posgrado en el exterior. Esta realidad ha cambiado radicalmente. Los docentes de ingreso reciente a la universidad, no cuentan con ninguna de estas ventajas. Esto ha incidido en una fragmentación de la autopercepción universitaria. Por una parte, los sectores que se formaron como universitarios en el período de auge, difícilmente comprenden el actual momento de crisis, en lo que respecta al agotamiento del modelo de universidad en el que se formaron, y en el que han desarrollado gran parte de su vida académica.

Por otra parte, los grupos administrativos y docentes del período de contracción, tampoco han logrado identificarse y comprometerse como es debido con el proyecto de modernización universitaria. Básicamente, debido a la inestabilidad laboral y a la escasa oportunidad de participar en los procesos de toma de decisiones, son indiferentes ante el cambio, que normalmente no les beneficia en forma significativa como trabajadores universitarios; esto se agrava si se considera que estos docentes normalmente trabajan tiempos parciales en otras instituciones, tanto públicas como privadas. Así, la inercia y la resistencia al cambio, que normalmente les perjudica, de los grupos que tienen poder para cambiar, sumada a la indiferencia de los trabajadores de tiempo parcial, hace que el clima para el cambio sea realmente incierto.

Estos hechos, entre los más obvios, han incidido en que la resistencia al cambio sea casi inmanejable y que la modernización, en muchas de sus áreas, pareciera que deberá esperar un mejor momento para darse e, incluso, que no va a darse nunca. La perspectiva de universidad que tienen esas dos generaciones -la del período de auge y la del período de contracción-, de lo que es la universidad, o de lo que debe ser, resulta incompatible con muchas de las demandas y presiones actuales sobre el sector. De hecho, como ejemplo de esto, el actual Rector de la U.C.R., Dr. Gabriel Macaya, en una entrevista publicada en el Semanario Universidad ${ }^{1}$, destaca, como uno de los principales problemas que ha encontrado en el primer año de su gestión, la inercia. Al respecto, apunta el Señor Rector que su administración está haciendo enormes esfuerzos por impulsar el cambio y por motivar una actitud constructiva frente a éste, a partir de un proceso complejo de información y retroalimentación, en torno a las políticas de cambio, con escasos resultados.

Los sectores y actores que disfrutaron de los privilegios del pasado no están dispuestos a perderlos. La misma legislación universitaria, como fue planteada en su oportunidad, hoy legitima la perpetuación de múltiples disfunciones administrativas y docentes. Como se ha podido ver, existen amplios sectores docentes y administrativos que no quieren o no les interesa el cambio, precisamente, porque ello implicaría la pérdida parcial o total de muchas de sus prerrogativas; o bien, la ganancia de pocas o ninguna ventaja.

Cuenta Toffler en su libro La empresa flexible, donde narra su experiencia como investigador en la AT \& T, a finales de la década de los años sesenta, cuando las grandes corporaciones empezaban a experimentar problemas de gestión importantes que, como norma, "las grandes organizaciones introducen cambios significativos sólo cuando se dan ciertas condiciones previas. Primero, debe haber enormes presiones externas. Segundo, debe existir gente dentro que esté muy descontenta con el orden existente. Y tercero, debe haber una alternativa coherente incorporada al plan, modelo o visión" (Toffler, 1995: 22-23). 
Siguiendo el análisis de Toffler, tan vigente hoy como hace treinta años, la universidad pública es una organización grande y compleja, donde sólo se han dado dos de las condiciones para el cambio. Hay enormes presiones externas, tanto a nivel gubernamental como social. Hay gente dentro de la universidad que está muy descontenta con el orden existente. Lo que hace falta es una alternativa coherente incorporada al plan, modelo o visión de la universidad pública necesaria para enfrentar los retos que implica el desarrollo nacional sostenible, con justicia social y calidad de vida crecientes. Sin embargo, ésto no va a ser posible si previamente no se hace una profunda transformación organizacional y cultural, donde se puedan consolidar las bases de un verdadero proceso de cambio.

Para René Lefort (1996), es indispensable hacer de nuevo las viejas preguntas ¿qué universidad, para quién y para qué?, tratando, en las respuestas que puedan darse a nivel institucional, de sacar el mayor provecho para nuestros países. Y algo que se debe tener muy claro, pese al discurso neoliberal, es que no es lo mismo ir detrás del mercado, que ir en pos del desarrollo nacional.

La teoría administrativa contemporánea, en palabras de los pensadores más representativos de las propuestas del cambio de paradigma, como Drucker (1995), Porter (1996) y Toffler (1995, 1996); así como importantes pensadores contemporáneos en filosofía política y sociología, como Offe (1990), Bell (1994), Giddens (1994) y Habermas (1993), coinciden en que la época de las organizaciones burocráticas de la era industrial, que alcanzaron su período de madurez en la primera mitad del siglo XX, caracterizadas por ser mecanicistas, jerárquicas, e inflexibles, y por ser diseñadas para la toma de decisiones repetitivas, han dejado de ser útiles. La organización del futuro tiende a ser horizontal, formada de pequeños componentes, flexible, y altamente participativa en los procesos de toma de decisiones y de asignación de responsabilidades. También, apuntan estos teóricos que el cambio en la época actual es más que un cambio en la estructura y funciones organizacionales públicas o privadas; afirman que es un cambio de civilización consecuencia de la revolución informática, las telecomunicaciones y del uso intensivo del conocimiento en el proceso productivo. Por esto, señalan que es normal que haya tanta confusión y dificultad para orientar el cambio. En todo caso, la necesidad de cambio es real, y el descontento interno y externo con nuestras universidades, son también reales. El cambio es cada vez más urgente.

Por lo expuesto hasta aquí, se hará una propuesta para una agenda de prioridades en torno a la modernización de la gestión universitaria pública, sin pretender con ello ni agotar el tema, ni cubrir todas sus áreas y complejidad. 


\section{Agenda de prioridades para la modernización de la gestión universitaria}

\author{
Fines y principios de la universidad pública: \\ 1. Revisar la misión de la universidad. \\ 2. Considerar a la universidad pública como proyec- \\ to político prioritario para el desarrollo nacional. \\ 3. Democratizar la universidad. \\ 4. Garantizar la autonomía interna. \\ 5. Redefinir las relaciones Gobierno-Universidad, \\ Universidad-sociedad.
}

Gestión universitaria:

1. Modernizar la gestión universitaria a nivel de estructura y legislación internas.

2. Sustituir el modelo administrativo tradicional por el estratégico.

3. Compatibilizar la estructura organizacional con los objetivos de eficiencia, eficacia y calidad.

4. Desarrollar a nivel estratégico los procesos de reclutamiento, inserción y contratación de personal docente y administrativo.

5. Implementar estrategias para el mejoramiento de las condiciones laborales de los docentes interinos.

6. Crear mecanismos de evaluación permanente de la gestión universitaria en todos sus ámbitos: acción social, docencia, investigación, vida estudiantil y administración. Crear mecanismos de control políticos, académicos y administrativos permanentes, a fin de garantizar la autonomía al interior y al exterior de la institución universitaria, como pilar de la práctica universitaria misma; es decir, a fin de asegurar los requisitos de libertad e igualdad necesarios para que haya pensamiento crítico, constructivo, creativo e innovador.

8. Profesionalizar la administración universitaria.

9. Desarrollar e incrementar la capacidad gerencial.

10. Orientar los recursos humanos, financieros y de infraestructura al logro de la calidad.

11. Crear mecanismos para el desarrollo de estrategias para la obtención de fuentes de financiamiento no gubernamentales.
Calidad de la educación:

1. Implementar una política de evaluación curricular, a fin de revisar los planes de estudio de todas las carreras que se imparte en la U.C.R., para analizar su grado de pertinencia con respecto al desarrollo actual de cada disciplina.

2. Eliminar la tendencia neoliberal educativa, que promueve el acortamiento de las carreras eliminando las áreas de formación integral y humanística, y de cultura general de los planes de estudio.

3. Crear políticas y proyectos concretos para integrar los tres niveles educativos públicos.

4. Crear mecanismos para la integración del sector universitario público, a fin de hacer un mejor uso de los recursos humanos, financieros y de infraestructura con que cuenta el país, a nivel global, en el sector terciario.

Mejoramiento continuo de la eficiencia, la eficacia y la calidad:

1. Implementar políticas de evaluación permanentes.

2. Fortalecer el vínculo entre la universidad y el sector productivo.

3. Implementar un sistema de administración universitaria moderno.

4. Elevar la capacidad tecnológica.

5. Desarrollar y potenciar el vínculo entre la universidad y la sociedad por medio de políticas de comunicación debidamente planificadas, a través de los medios de difusión propios. Esto es, crear condiciones para que los periódicos, canales de televisión y frecuencias radiales de las universidades cumplan con la misión universitaria de difundir la cultura y el conocimiento, promoviendo espacios adecuados para el desarrollo de un pensamiento crítico, reflexivo y creativo necesario para el fortalecimiento de una sociedad democrática. 
Fines y principios de la universidad pública

\section{Problemática}

Resulta indispensable una revisión profunda de la misión de la universidad pública, debido al cambio radical que ha sufrido el sistema económico mundial. El impacto de esta nueva estructura económica, basada en el uso intensivo del conocimiento en los procesos productivos, hace que éste se convierta en el principal factor productivo. Por primera vez en la historia de la humanidad, aquellas regiones o países rezagados en los niveles de desarrollo, tienen la oportunidad de crear, con una inversión adecuada del presupuesto nacional, condiciones de eficiencia, eficacia y calidad, que les permita redimensionar sus ventajas comparativas a nivel mundial. Además, es un hecho que los países pequeños, si no se unen en un esfuerzo nacional y regional, difícilmente saldrán adelante ante las actuales reglas del juego económico mundial, donde los acuerdos del GATT, por ejemplo, permiten políticas proteccionistas solapadas en los países desarrollados, pero no en los pobres. El juego de poder del nuevo orden mundial, como suele ocurrir, vuelve a desfavorecer a los débiles.

Dadas estas razones, es fundamental considerar la educación en general y, en particular, la superior como el principal proyecto político nacional y regional.

Para lograr que este proyecto se convierta en realidad, es necesario que la sociedad como un todo reconozca la importancia del proyecto. Tal reconocimiento depende de que la universidad misma, como institución estratégica, reconozca su misión y geste los procesos de cambio internos que hacen falta para el logro de tal propósito. En este sentido, la democratización de la universidad es condición necesaria, aunque no suficiente, para el cambio.

Otro aspecto fundamental para la redefinición del rol de la universidad pública, es el logro de políticas de cooperación nacional, regional e internacional, para el mejoramiento continuo de la calidad educativa. Este sería uno de los principales aspectos a lograr en lo que concierne a las nuevas formas de relación gobierno-universidad-sociedad .

\section{Propuesta de solución}

En el largo plazo:

1. Crear políticas para una integración del sistema educativo público nacional, que permitan articular los tres sectores, de tal modo que pueda asegurarse la calidad, pertinencia y equidad de la educación en forma permanente. En este sentido, se pueden crear alianzas estratégicas en el sector público.

2. Crear políticas para la integración del sistema universitario público, que permitan la formulación de planes de desarrollo estratégico coherentes con las necesidades del país, en cada momento histórico. Esto permitirá evitar duplicidades, uso inadecuado de recursos, o esquemas de desarrollo sin orientación estratégica.

3. Desarrollar mecanismos para el logro de la integración de la educación superior pública centroamericana a través del CSUCA, a fin de crear opciones para la cooperación financiera, técnica y académica, así como para el intercambio de docentes y estudiantes. Esto implicaría, además, el desarrollo de políticas de acreditación y reconocimiento de grados académicos en la región.

En el mediano plazo:

1. Abrir espacios para que los distintos sectores, tanto internos como externos, a la universidad participen en un foro bienal para el mejoramiento de la educación superior nacional.

2. Presentar al CSUCA la iniciativa de abrir espacios para el desarrollo de foros centroamericanos orientados al mejoramiento de la educación superior en la región, a realizarse en plazos no mayores de tres años, tomando como sede a cada uno de los distintos países, en forma consecutiva. Esto, además, permitiría un mejor conocimiento y comprensión entre nuestros pueblos, así como más y mejores alternativas para un desarrollo económico sostenible en la región. La unión regional es una de las principales características de la globalización.

En el corto plazo:

1. Impulsar y orientar procesos de revisión de la misión universitaria a nivel de cada unidad académica.

2. Convocar a la comunidad universitaria a un foro de reflexión sobre la misión de la universidad pública, ante los retos del nuevo orden económico, político y social de la civilización globalizada, después de concluidas las jornadas de revisión de la misión de la universidad. Precisamente, el insumo de este foro de reflexión serían los datos obtenidos en tales jornadas. 


\section{Gestión Universitaria}

\section{Problemática}

Uno de los principales problemas de la universidad pública es el desgaste del modelo organizacional de corte burocrático, altamente politizado. Las típicas disfunciones de la burocracia como: lentitud, ineficiencia, ineficacia, despersonalización de las relaciones, internalización de las normas, exceso de formalismo y papeleo, resistencia al cambio, exhibición de señales de autoridad, categorización de las decisiones, excesiva conformidad, y pobre calidad de los servicios, que genera una gran frustración y descontento en los destinatarios del mismo, son hechos cotidianos en nuestras universidades.

Además, las ventajas conferidas a la organización burocrática, como: el carácter legal de las normas, el carácter formal de las comunicaciones, la división rígida del trabajo, la impersonalidad en las relaciones laborales, la jerarquización de la autoridad, los procedimientos y tareas rutinarios; resultan contraproducentes ante la nueva realidad social, económica y laboral de las organizaciones modernas. A nuestro parecer, las únicas ventajas vigentes de la burocracia son la especialización de la administración y la profesionalización.

Otros aspectos fundamentales asociados con la estructura organizacional y funcional de la universidad, son las deficientes condiciones laborales impuestas a las nuevas generaciones de trabajadores, tanto docentes como administrativos.

La creación de una cultura organizacional de calidad, abierta al cambio y a la evaluación permanente, es condición necesaria para el desarrollo del nuevo modelo universitario.

La profesionalización de la gestión universitaria resulta indispensable, a fin de que se creen políticas para el desarrollo y mejoramiento de la capacidad gerencial. La complejidad y diversidad de las acciones administrativas de una institución de educación superior, demanda una administración universitaria altamente capacitada. Ya no es posible que personas sin conocimientos en administración, asuman cargos directivos.

Otro aspecto fundamental para el logro de la modernización en la gestión universitaria es potenciar las fuerzas que promueven el cambio. En este sentido, el liderazgo ejercido por las autoridades universitarias, debe ir orientado, precisamente, a la promoción de las iniciativas de cambio.

\section{Propuesta de solución}

En el largo plazo:

1. Crear mecanismos de evaluación del desempeño en todas las áreas de la universidad.

2. Desarrollar un sistema de incentivos donde la docencia, la investigación y la extensión, sean consideradas desde una perspectiva clara en función de su relevancia en el desarrollo de la universidad y de la sociedad. Particularmente, la docencia y la extensión han sido mal ponderadas en el actual sistema de ascenso en Régimen Académico.

3. Crear un Instituto de Investigaciones para el desarrollo de la Educación Superior, a fin de contar con producción de conocimiento pertinente para el mejoramiento continuo de la universidad pública en general, y de la Universidad de Costa Rica en particular. Además, como una de las principales funciones de este instituto, estaría desarrollar planes para la formación, capacitación y actualización de personal universitario con interés de ocupar cargos directivos. Además, como política, la universidad debe solicitar esta formación como requisito para ocupar dichos cargos. La Maestría en Administración Universitaria, constituiría un importante medio para proveer el recurso humano necesario para el funcionamiento general del instituto.

4. Plantear la iniciativa al CSUCA para la propuesta de la creación de institutos homólogos en cada uno de los países centroamericanos.

En el mediano plazo:

1. Implementar y operacionalizar los proyectos para el logro de las estrategias orientadas al cambio, obtenidas por medio del proceso de autoevaluación.

2. Revisar la vigencia de la legislación y normativa universitaria actual, a fin de detectar posibles disfunciones e, incluso, la obsolescencia de las mismas.

3. Proponer a las unidades académicas y demás dependencias universitarias, el desarrollo de mecanismos de evaluación del desempeño docente-administrativo, desde la lógica gerencial contemporánea. 


\begin{tabular}{|c|c|c|}
\hline \multicolumn{3}{|c|}{ Gestión Universitaria } \\
\hline $\begin{array}{l}\text { Problemática } \\
\text { Debido a la inercia que impera en la universidad, el li- } \\
\text { derazgo más eficaz sería el de tipo participativo y au- } \\
\text { toadministrado (Stonner, 1992: 527), basado en el tra- } \\
\text { bajo de equipo por áreas académicas y administrativas, } \\
\text { a nivel de coordinadores de área, equipos de apoyo, } \\
\text { equipos técnicos y equipos operativos. Un fuerte lide- } \\
\text { razgo participativo es fundamental para potenciar el } \\
\text { cambio en una institución de educación superior. } \\
\text { Potenciar las fuerzas del cambio supone fomentar la par- } \\
\text { ticipación (Davis y Newstrom, 1994: 343). En este senti- } \\
\text { do, una de las principales metas de los equipos coordi- } \\
\text { nadores es orientar las acciones a fin de vencer las ba- } \\
\text { rreras lógicas, psicológicas y sociológicas propias de ca- } \\
\text { da grupo o unidad de trabajo, empleando una lógica de } \\
\text { desarrollo de sinergia y estrategias de negociación ganar- } \\
\text { ganar. } \\
\text { Finalmente, otra de las principales tareas de la gestión } \\
\text { universitaria es evaluar las posibilidades de potenciar } \\
\text { acciones tendientes al desarrollo de programas para la } \\
\text { obtención de recursos financieros no gubernamentales. } \\
\text { En este sentido, deben promoverse las alianzas estraté- } \\
\text { gicas con instituciones públicas y privadas, tanto nacio- } \\
\text { nales como internacionales, e incrementar las opciones }\end{array}$ & $\begin{array}{l}5 . \\
6 .\end{array}$ & $\begin{array}{l}\text { Propuesta de solución } \\
\text { corto plazo: } \\
\text { Consolidar una comisión interdisciplinaria para } \\
\text { la gerencia del proceso de modernización, com- } \\
\text { puesto por personas comprometidas con el } \\
\text { cambio, con trayectoria universitaria. Esta comi- } \\
\text { sión estará presidida por el Señor Rector y por } \\
\text { los cinco Vicerrectores, que presidirán, a su vez, } \\
\text { subcomisiones técnicas para cada área de la } \\
\text { gestión universitaria; es decir, para las áreas de } \\
\text { Docencia, Investigación, Acción Social, Admi- } \\
\text { nistración y Vida Estudiantil. Las subcomisiones } \\
\text { técnicas deben estar conformadas por el perso- } \\
\text { nal interdisciplinario requerido para el proceso, } \\
\text { según las especificidades de cada área. } \\
\text { Coordinar acciones con las dependencias uni- } \\
\text { versitarias que pueden colaborar en forma } \\
\text { significativa en el desarrollo de los procesos } \\
\text { de autoevaluación y modernización. } \\
\text { Garantizar que el proceso de autoevaluación } \\
\text { esté orientado, con base en los logros del foro } \\
\text { de reflexión universitaria. Además, como un } \\
\text { elemento positivo para el curso de este proce- } \\
\text { so, está el que la U.C.R. ya cuenta con expe- } \\
\text { riencias piloto de autoevaluación, dirigidas por } \\
\text { el Centro de Evaluación Académica, como par- } \\
\text { te del proyecto Columbus. } \\
\text { Desarrollar sistemas de información específi- } \\
\text { cos para el proceso de modernización univer- } \\
\text { sitaria. Esto es fundamental, ya que tanto el } \\
\text { conocimiento como la transparencia en lo que } \\
\text { respecta a los objetivos, metas y logros del } \\
\text { proceso, así como de su desarrollo, son indis- } \\
\text { penursos propios a través de la venta de bie- } \\
\text { nes o servicios. } \\
\text { lncluir en el proceso de autoevaluación la va- } \\
\text { activa de los distintos sectores universitarios. } \\
\text { De lo contrario, el proceso pierde legitimidad } \\
\text { y se convierte en otro intento de cambio que } \\
\text { no va más allá de las buenas intenciones. } \\
\text { Iniciar procesos de autoevaluación en todas } \\
\text { las dependencias universitarias. } \\
\text { Incluir en el proceso de autoevaluación la va- } \\
\text { loración de proyectos para el desarrollo de } \\
\text { alianzas estratégicas con el sector público y } \\
\text { rentel nacional e internacional, }\end{array}$ \\
\hline
\end{tabular}


Calidad de la educación

\section{Problemática}

La crisis de calidad que sufre la educación pública en general, y en particular la universidad, ha generado una importante discusión a nivel nacional e internacional. La UNESCO, ha hecho enormes esfuerzos con el propósito de generar iniciativas para el mejoramiento de la calidad.

Precisamente, uno de los problemas centrales de la calidad en educación, es que esta deviene un concepto complejo, debido a que involucra varios aspectos a nivel sistémico, de orden pedagógico, gerencial e institucional (Puiggrós y Krotsch, 1994: 185 y ss). Por este motivo, la calidad en educación supone la consideración de la calidad de vida, en términos de pertinencia, equidad e impacto social.

La educación se ha convertido en uno de los principales insumos para el desarrollo, debido a que el conocimiento es el principal factor productivo. En este sentido, la educación se convierte en área estratégica para el desarrollo de los países del Tercer Mundo. Por esto, como se ha señalado, siguiendo a Tedesco, la educación constituye un asunto de supervivencia para América Latina.

Debido a lo expuesto, se reitera que el principal proyecto político de la región latinoamericana es el desarrollo de una educación de alta calidad, a fin de ofrecer mejores oportunidades de vida a nuestros pueblos. De la inversión estatal y social que se haga en ella, dependerá, en gran medida, el futuro económico, social y cultural de América Latina.

Otro punto central a considerar es la articulación del sistema educativo, a fin de que los tres sectores operen en forma coordinada y coherente. Esto es, que la secundaria no deba remediar problemas de primaria, y que la universidad no deba remediar problemas de secundaria. Si esto se lograra, nuestra población estudiantil llegaría con más bases teórico-prácticas y mejores condiciones para el aprendizaje orientado a la comprensión, al análisis y a la investigación. Esto redundaría en un mejor aprovechamiento de la educación superior, una menor deserción y un más alto rendimiento académico.

\section{Propuesta de solución}

En el largo plazo:

1. Integrar el sistema de educación superior a nivel regional.

2. Integrar el sistema de educación superior pública nacional.

3. Integrar el sistema de educación nacional. La universidad debe hacer un mayor aporte para el mejoramiento continuo de la calidad educativa en los tres sectores. La fragmentación sólo ha ocasionado que cada uno de ellos arrastre las deficiencias del anterior. Por esto, la universidad resulta la más perjudicada, en la medida que debe asumir las deficiencias de la formación secundaria, básica para un buen aprovechamiento de la educación superior. En este sentido, las acciones de la Facultad de Educación resultan medulares, ya que es la que forma los educadores y administradores educativos de los sectores básicos, medios y universitarios.

En el mediano plazo:

1. Implementar políticas de evaluación permanente de los planes de estudio, a fin de mantenerlos actualizados.

2. Crear programas de capacitación permanente para el sector educativo nacional.

3. Abrir espacios para la discusión permanente de la educación nacional. Como foros, congresos, seminarios, talleres, entre otros.

En el corto plazo:

1. Iniciar un proceso de revisión curricular en todas las unidades académicas, a fin de evaluar la pertinencia y calidad de la oferta educativa.

2. Fortalecer el área docente, creando mejores condiciones laborales para el personal docente.

3. Implementar programas permanentes de profesionalización de la docencia universitaria.

4. Fortalecer la investigación y la acción social, como complementos insustituibles de la docencia. 


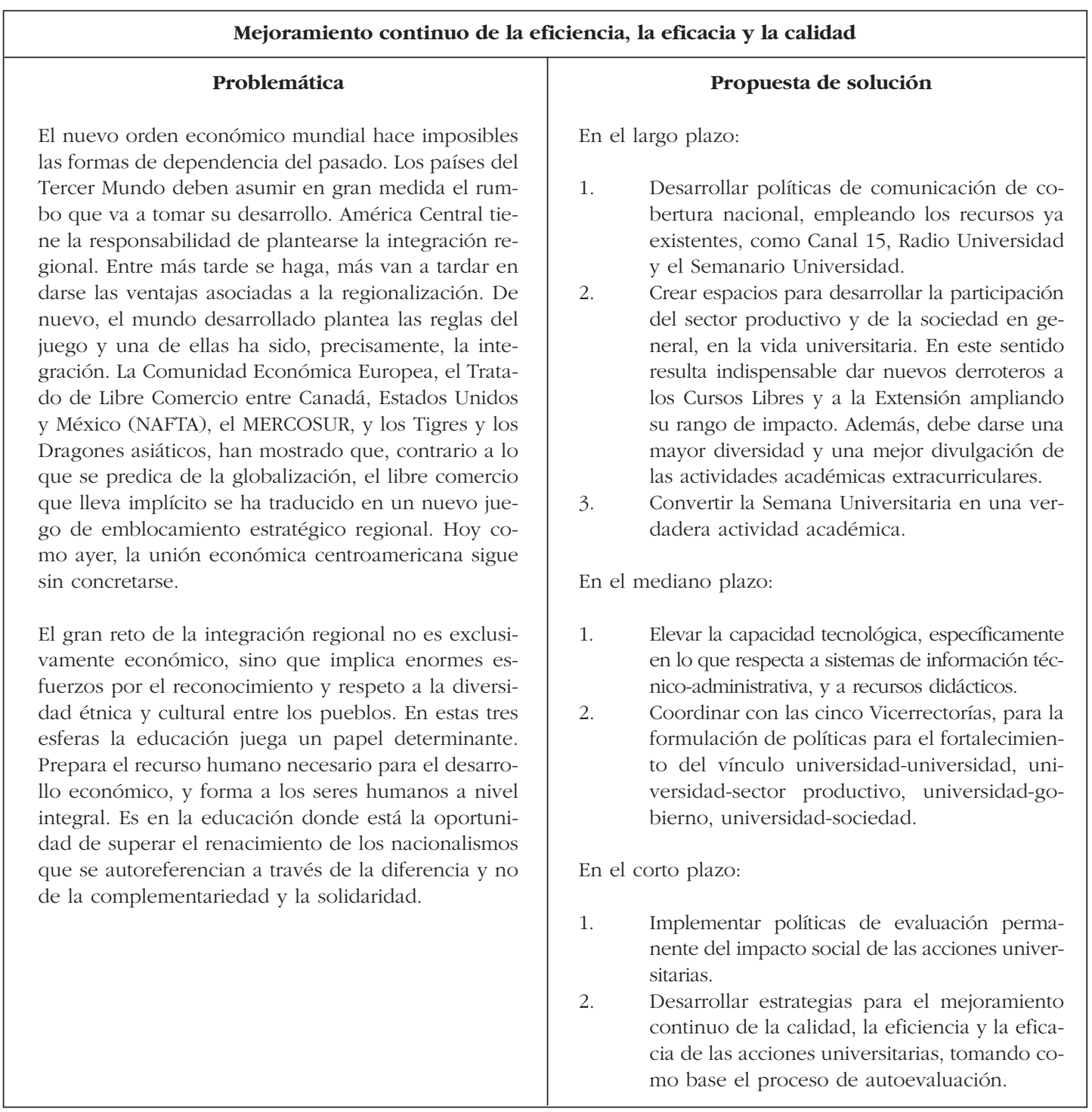

\section{Conclusiones}

Es evidente que el cambio en la gestión universitaria supone la participación y el consenso de toda la comunidad universitaria. Sin embargo, para que el cambio se geste, resulta indispensable una clara voluntad política, por parte de las autoridades universitarias, para crear las condiciones necesarias para la participación y la concertación. Un liderazgo fuerte y eficaz es indispensable para propiciar el cambio.
Una de las acciones administrativas que más obstaculizan el cambio es, precisamente, la inacción. El implementar acciones tendientes al logro del cambio es el primer paso del cambio. Este es el principal reto de la actual administración.

Lo más desalentador de la situación actual de la universidad es que hay muchas propuestas de cambio en el papel, pero no se ven acciones orientadas a su puesta en marcha. Un adecuado equilibrio entre teoría y práctica, es necesario para que el proceso de 
modernización sea llevado a cabo. Esto evidencia cuán ajena ha Estado la universidad de sí misma, cuánto se ha distanciado de sus propósitos originales, y cuánto ha cambiado.

De una clara voluntad política por el cambio va a depender la puesta en marcha de una modernización auténtica. Pero también va a depender de los espacios de discusión y participación reales que se pongan a disposición de los distintos actores. Capitalizar los errores es parte del proceso de cambio, de igual modo que lo es el capitalizar los aciertos. Esto sólo es posible a través de la vivencia de acciones concretas. Dice el refrán que no hay nada más práctico que una buena teoría; sin embargo, la mejor teoría es irrelevante si no se pone en práctica.

\section{Nota}

1. Inercia frena el cambio. (1997, Julio) Universidad, pp. 6-7.

\section{Referencias bibliográficas}

Arroyo, Juan Antonio. et al. Consecuencias de los Programas de Ajuste Estructural en el sector educativo. San José: Universidad de Costa Rica, Tesis de Maestría en Administración Pública, sin publicar.

Bell, Daniel. El advenimiento de la sociedad post-industrial. Madrid: Alianza Editorial, 1994.

Drucker, Peter. Gerencia para el futuro. Colombia: Norma, 1995.

Giddens, Anthony. La estructura de clases en las sociedades avanzadas. Madrid: Alianza Editorial, 1994.

Habermas, Jürgen. El discurso filosófico de la modernidad. Madrid: Taurus, 1993.

Jofré, Arturo. La Universidad en América Latina. Desafíos y estrategias para las próximas décadas. Cartago: Editorial Tecnológica de Costa Rica, 1994.

Offe, Claus. Contradicciones en el Estado de Bienestar. 1era. edición. Madrid: Alianza Editorial, 1990.

Porter, Michael. Estrategia competitiva. México: CECSA, 1996.

Toffler, Alvin. La empresa flexible. Barcelona: Plaza \& Janés, 1995. La Tercera Ola. Barcelona: Plaza \& Janés, 1996.

Tünnermann, Carlos. Ensayos sobre teoría de la universidad. Managua: Vanguardia, 1990.

Calidad y evaluación de la educación superior. Conferencia dictada en el Primer Foro Regional: "La Universidad Centroamericana hacia el Tercer Milenio". San José, C.R.: 17 y 18 de Octubre de 1996.

CSUCA. Memoria del IV Congreso Universitario Centroamericano. San José, C. R.: EDUCA, 1996.

II Plan para la Integración Regional de la Educación Superior Centroamericana. PIRESC II. San José, C. R.: EDUCA, 1996.

UNESCO. Reunión Internacional de Reflexión sobre los nuevos roles de la Educación Superior a nivel mundial: el caso de América Latina y del Caribe, futuro y escenarios deseables. Nuevos contextos y perspectivas. Volumen I. Caracas: UNESCO/CRESALC, 1991.

Universidad de Costa Rica. Serie de diagnósticos. Panorama cuantitativo. Oficina de Planificación Universitaria, 1995. 